\title{
Integrals of Special Functions and Umbral Methods
}

\section{G. Dattoli ${ }^{1}$ - B. Germano ${ }^{2}$ S. Licciardi ${ }^{1}$ (D) $\cdot$ M. R. Martinelli ${ }^{2}$}

Accepted: 9 May 2021 / Published online: 4 June 2021

(C) The Author(s) 2021

\begin{abstract}
We derive integrals of combination of Gauss and Bessel functions, by the use of umbral techniques. We show that the method allows the possibility of pursuing new and apparently fruitful avenues in the theory of special functions, displaying interesting links with the theory and the formalism of integral transforms.
\end{abstract}

Keyword Umbral calculus · Operator theory · Bessel function · Special functions · Integrals $\cdot$ Integral transforms

\section{Introduction}

Methods of algebraic and umbral nature possess, among the other advantages, the undoubtful merit of simplifying practical computational issues. In a numbers of previous papers [1-7] it has been established that the one of the possible umbral images of a Bessel function is a Gaussian. This statement can be profitably exploited in the present context, provided we establish some reference tools $[6,8]$.

Definition 1 The function

$$
\varphi(v):=\varphi_{v}=\frac{1}{\Gamma(v+1)}, \quad \forall v \in \mathbb{R} .
$$

defines the Umbral "Vacuum" function or simply vacuum.

\section{S. Licciardi}

silvia.licciardi@enea.it; silviakant@gmail.com

G. Dattoli

pinodattoli@enea.it

B. Germano

bruna.germano@sbai.uniroma1.it

M. R. Martinelli

martinelli@dmmm.uniroma1.it

1 ENEA - Frascati Research Center, Via Enrico Fermi 45, 00044 Frascati, Rome, Italy

2 Department of Methods and Mathematic Models for Applied Sciences, University of Rome, La Sapienza, Via A. Scarpa, 14, 00161 Rome, Italy 
Definition 2 We introduce the operator $\hat{c}$ called "umbral",

$$
\hat{c}:=e^{\partial_{z}},
$$

as the vacuum shift operator, with $z$ the domain's variable of the function on which the operator acts.

Theorem 1 The umbral operator $\hat{c}^{\nu}, \forall v \in \mathbb{R}$, is represented by the action on the vacuum $\varphi_{0}$ such that ${ }^{1}$

$$
\hat{c}^{v} \varphi_{0}=\frac{1}{\Gamma(v+1)} .
$$

Proof $\forall v \in \mathbb{R}$, by using Definition 2, we get

$\hat{c}^{v} \varphi_{0}=\left.\hat{c}^{v} \varphi(z)\right|_{z=0}=\left.e^{v \partial_{z}} \varphi(z)\right|_{z=0}=\left.\varphi(z+v)\right|_{z=0}=\left.\frac{1}{\Gamma(z+v+1)}\right|_{z=0}=\frac{1}{\Gamma(v+1)}$.

Through these premises, we define the correspondence quoted below.

Proposition $1 \forall x, v \in \mathbb{R}$, the umbral image of a 0-order Bessel function is a Gaussian function

$$
J_{0}(x)=e^{-\hat{c}\left(\frac{x}{2}\right)^{2}} \varphi_{0} .
$$

Proof $\forall x, v \in \mathbb{R}$, let

$$
u(x)=e^{-\hat{c}\left(\frac{x}{2}\right)^{2}} \varphi_{0},
$$

the relevant series expansion proofs that $u(x)=J_{0}(x)$, indeed

$$
u(x)=e^{-\hat{c}\left(\frac{x}{2}\right)^{2}} \varphi_{0}=\sum_{r=0}^{\infty} \frac{(-\hat{c})^{r}}{r !}\left(\frac{x}{2}\right)^{2 r} \varphi_{0}=\sum_{r=0}^{\infty} \frac{(-1)^{r}}{(r !)^{2}}\left(\frac{x}{2}\right)^{2 r}=J_{0}(x) .
$$

It is accordingly evident that we can exploit all the wealth of properties of Gaussian function to deal with those of Bessel, this has already been discussed in previous papers [3] and won't be repeated here.

In order to summarize the method we are going to exploit, we consider the following Example.

Example 1 We prove that the previous prescription allows to reduce the evaluation of the integral

$$
I_{J}(a, b)=\int_{-\infty}^{\infty} J_{0}\left(2 \sqrt{a x^{2}+b x}\right) d x, \quad \forall a, b \in \mathbb{R}: a x^{2}+b x>0
$$

to a straightforward Gaussian integration. We note indeed that

$$
I_{J}(a, b)=\int_{-\infty}^{\infty} e^{-\hat{c}\left(a x^{2}+b x\right)} d x \varphi_{0}
$$

\footnotetext{
1 See ref. [6] for a rigorous treatment of the umbral technique.
} 
and, according to the well-known integral identity

$$
I_{G}(a, b)=\int_{-\infty}^{\infty} e^{-\left(a x^{2}+b x\right)} d x=\sqrt{\frac{\pi}{a}} e^{\frac{b^{2}}{4 a}}
$$

we can conclude that

$$
I_{J}(a, b)=I_{G}(\hat{c} a, \hat{c} b) \varphi_{0}=\sqrt{\frac{\pi}{\hat{c} a}} e^{\frac{\hat{c} b^{2}}{4 a}} \varphi_{0} .
$$

In deriving Eq. (11) we have treated the umbral operator as an ordinary algebraic quantity. It is now necessary to manipulate Eq. (11) to get a meaningful result, we should indeed provide a non-formal expression for the operator function on the r.h.s. of Eq. (11). To this aim we expand in series the exponential on the r.h.s. of (11) and by using the properties of the $\hat{c}$-operator we find

$$
I_{J}(a, b)=\sqrt{\frac{\pi}{a}} \sum_{r=0}^{\infty} \frac{1}{r !}\left(\frac{b}{2 \sqrt{a}}\right)^{2 r} \hat{c}^{r-\frac{1}{2}} \varphi_{0}=\sqrt{\frac{\pi}{a}} \sum_{r=0}^{\infty} \frac{1}{r ! \Gamma\left(r+\frac{1}{2}\right)}\left(\frac{b}{2 \sqrt{a}}\right)^{2 r} .
$$

The series on this last equation can easily be recognized as a Bessel function and therefore we end up with

$$
I_{J}(a, b)=\sqrt{\frac{\pi}{a}} \sqrt{\frac{b}{2 \sqrt{a}}} I_{-\frac{1}{2}}\left(\frac{b}{\sqrt{a}}\right)
$$

where $I_{v}(x)$ is a modified Bessel of first kind [9].

This result, not straightforwardly achievable by standard means, is a naïve consequence of the previously outlined procedure.

The example we have presented is sufficient to clarify the essential features of the methods we are going to use in the following parts of the paper. We will see below that, by stretching the formalism, we can provide further and more interesting results.

\section{Integrals and Non Gaussian Umbral Images}

Before entering the specific aspects of this section, we like to stress that the methods we are reporting here are useful and flexible but not unique. We will check the relevant usefulness by deriving well established forms obtained in the past $[10,11]$

Example 2 We consider the further example

$$
I_{J, G}(a, b)=\int_{-\infty}^{\infty} J_{0}(2 \sqrt{a} x) e^{-b x^{2}} d x
$$

which, according to the previous formalism, can be written as

$$
I_{J, G}(a, b)=\int_{-\infty}^{\infty} e^{-\hat{c} a x^{2}} e^{-b x^{2}} d x \varphi_{0}=I_{G}(\hat{c} a+b, 0) \varphi_{0}
$$


and, therefore, we end up with ${ }^{2}$

$$
I_{J, G}(a, b)=\sqrt{\frac{\pi}{b}} \frac{1}{\sqrt{1+\frac{a}{b}} \hat{c}} \varphi_{0}=\sqrt{\frac{\pi}{b}} \frac{1}{\Gamma\left(\frac{1}{2}\right)} \int_{0}^{\infty} e^{-s} s^{-\frac{1}{2}} e^{-\frac{a s}{b} \hat{c}} d s \varphi_{0} .
$$

Therefore, after noting that [6]

$$
\begin{aligned}
& \int_{0}^{\infty} e^{-s} s^{-\frac{1}{2}} e^{-x s \hat{c}} d s \varphi_{0}=\sum_{r=0}^{\infty} \frac{\Gamma\left(r+\frac{1}{2}\right)(-x)^{r}}{r !^{2}}=c_{0,-\frac{1}{2}}^{(1)}(x), \\
& c_{\mu, \alpha}^{(v)}(x)=\sum_{r=0}^{\infty} \frac{\Gamma(v r+\alpha+1)}{r ! \Gamma(r+\mu+1)}(-x)^{r}, \quad \forall \alpha, \mu, v \in \mathbb{R}^{+}, \forall x \in \mathbb{R}
\end{aligned}
$$

we find

$$
I_{J, G}(a, b)=\frac{1}{\sqrt{b}} c_{0,-\frac{1}{2}}^{(1)}\left(\frac{a}{b}\right)
$$

We will comment on the properties of the auxiliary function $c_{\mu, \alpha}^{(v)}(x)$ [6] in the concluding section.

The use of a Gaussian function as umbral image is useful but not unique. The umbral method has allowed to reduce a higher transcendental function, like a Bessel function, to an ordinary trascendental, like a Gaussian. By following the same "down-grading" criterion we can use rational functions as umbral image of exponentials. We note indeed that a Lorentzian function is the umbral image of the Gaussian function according to the identities

$$
e^{-a x^{2}}=\sum_{r=0}^{\infty} \frac{(-a)^{r}}{r !} x^{2 r}=\sum_{r=0}^{\infty}(-a \hat{c})^{r} x^{2 r} \varphi_{0}=\frac{1}{1+a \hat{c} x^{2}} \varphi_{0}
$$

We will exploit the above umbral restyling by deriving an integral involving Gaussian and Lorentzian functions.

Example 3 Let

$$
I_{L}(a, b)=\int_{-\infty}^{\infty} \frac{e^{-\frac{a x^{2}}{1+b x^{2}}}}{1+b x^{2}} d x, \quad \forall a, b \in \mathbb{R}^{+},
$$

2 We use the Laplace transform identity

$$
A^{-v}=\frac{1}{\Gamma(v)} \int_{0}^{\infty} s^{v-1} e^{-s A} d s
$$


the use of the outlined formalism, eventually yields ${ }^{3}$

$$
\begin{aligned}
I_{L}(a, b) & =\int_{-\infty}^{\infty} \frac{1}{\left(1+b x^{2}\right)\left(1+\hat{c} \frac{a x^{2}}{1+b x^{2}}\right)} d x \varphi_{0}=\int_{-\infty}^{\infty} \frac{1}{1+(b+a \hat{c}) x^{2}} d x \varphi_{0} \\
& =I_{L}(0, b+a \hat{c}) \varphi_{0}=\frac{\pi}{\sqrt{b+a \hat{c}}} \varphi_{0}=\frac{\sqrt{\pi}}{\sqrt{b}} c_{0,-\frac{1}{2}}^{(1)}\left(\frac{a}{b}\right) .
\end{aligned}
$$

The use of the same formalism yields $\forall b>0$

$$
\begin{aligned}
I_{E}(a, b) & =\int_{-\infty}^{\infty} \frac{e^{-\frac{a x}{1+b x^{2}}}}{1+b x^{2}} d x=\int_{-\infty}^{\infty} \frac{1}{1+a \hat{c} x+b x^{2}} d x \varphi_{0} \\
& =\sqrt{\frac{\pi}{b}} J_{0,-\frac{1}{2}}^{(2)}\left(\frac{a}{\sqrt{b}}\right), \\
J_{\mu, \nu}^{(\alpha)}(x) & =\sum_{r=0}^{\infty} \frac{\Gamma(r+v+1)}{r ! \Gamma(\alpha r+\mu+1)}\left(\frac{x}{2}\right)^{2 r+\mu}, \quad \forall \alpha, \mu, v \in \mathbb{R}^{+}, \forall x \in \mathbb{R} .
\end{aligned}
$$

We can now apply the method to more complicated functions.

Example 4 We consider indeed the case

$$
I_{L, J}(a, b)=\int_{-\infty}^{\infty} \frac{J_{0}\left(\frac{2 \sqrt{a} x}{\sqrt{1+b x^{2}}}\right)}{1+b x^{2}} d x, \quad \forall a, b \in \mathbb{R}^{+},
$$

which, according to our formalism, can be transformed into a $G-L$ integral and eventually into a $L$-integral. Accordingly we get

$$
I_{L, J}(a, b)=I_{L}(a \hat{c}, b) \varphi_{0}=\sqrt{\frac{\pi}{b}} c_{0,-\frac{1}{2}}^{(1)}\left(\frac{a \hat{c}}{b}\right) \varphi_{0}=\sqrt{\frac{\pi}{b}} \sum_{r=0}^{\infty} \frac{\Gamma\left(r+\frac{1}{2}\right)}{r !^{3}}\left(-\frac{a}{b}\right)^{r}
$$

where the series on the r.h.s. is a Humbert type Bessel function, to be commented in the concluding section. Furthermore, if we introduce a new umbral operator ${ }_{2} \hat{c}$ such that

$$
{ }_{2} \hat{c}^{r} \psi_{0}=\frac{1}{(\Gamma(r+1))^{2}},
$$

we end up with

$$
I_{L, J}(a, b)=\sqrt{\frac{\pi}{b}} \sum_{r=0}^{\infty} \frac{\Gamma\left(r+\frac{1}{2}\right)}{r !}\left(-\frac{a_{2} \hat{c}}{b}\right)^{r} \psi_{0}=\frac{\pi}{\sqrt{b+a_{2} \hat{c}}} \psi_{0}=I_{L}\left(0, b+a_{2} \hat{c}\right) \psi_{0} .
$$

The technicalities, we have displayed in this section, yield an idea of the usefulness of the methods we have foreseen. Further comments and examples, corroborating the previous results, will be presented in the concluding section.

${ }^{3}$ We remind that

$$
\int_{-\infty}^{\infty} \frac{1}{1+a x^{2}} d x=\frac{\pi}{\sqrt{a}}, \forall a \in \mathbb{R}^{+} .
$$




\section{Final Comments}

The results of the previous sections, albeit supported by the check of the relevant correctness, including comparison with known cases and numerical analysis, are hampered by the absence of mathematical rigor. In Ref. [6] a great deal of attention has been devoted to a more appropriate formulation. In the following we will extend the method by including the recourse to families of Special Polynomials.

Example 5 We can provide further elements of discussion by setting

$$
J_{0}\left(2 \sqrt{\alpha x+\beta x^{2}}\right)=\frac{1}{1+{ }_{2} \hat{c}\left(\alpha x+\beta x^{2}\right)} \psi_{0}, \quad \forall \alpha, \beta \in \mathbb{R}^{+} .
$$

We now remind that the two variable Cebyshev polynomials of second kind [9],

$$
U_{n}(x, y)=(-1)^{n} \sum_{r=0}^{\left\lfloor\frac{n}{2}\right\rfloor} \frac{(n-r) ! x^{n-2 r}(-y)^{r}}{(n-2 r) ! r !}, \forall x, y \in \mathbb{R}, \forall n \in \mathbb{N},
$$

are specified by the generating function

$$
\sum_{n=0}^{\infty} t^{n} U_{n}(x, y)=\frac{1}{1+x t+y t^{2}}, \quad \operatorname{Re}\left(1+x t+y t^{2}\right)>0 .
$$

According to Eq. (27) we can conclude that

$$
J_{0}\left(2 \sqrt{\alpha x+\beta x^{2}}\right)=\sum_{n=0}^{\infty} x^{n} U_{n}(\alpha \hat{b}, \beta \hat{b}) \psi_{0} .
$$

and therefore, by noting that

$$
\begin{aligned}
U_{n}(\alpha \hat{b}, \beta \hat{b}) \psi_{0} & =(-1)^{n} \sum_{r=0}^{\left\lfloor\frac{n}{2}\right\rfloor} \frac{(-1)^{r}(n-r) ! \alpha^{n-2 r} \beta^{r}}{(n-2 r) ! r !} \hat{b}^{n-r} \psi_{0} \\
& =(-1)^{n} \sum_{r=0}^{\left\lfloor\frac{n}{2}\right\rfloor} \frac{(-1)^{r} \alpha^{n-2 r} \beta^{r}}{(n-2 r) !(n-r) ! r !},
\end{aligned}
$$

we find

$$
\begin{aligned}
& J_{0}\left(2 \sqrt{\alpha x+\beta x^{2}}\right)=\sum_{n=0}^{\infty} x_{2}^{n} U_{n}(\alpha, \beta), \quad \forall x, \alpha, \beta \in \mathbb{R} \\
& { }_{2} U_{n}(\alpha, \beta):=(-1)^{n} \sum_{r=0}^{\left\lfloor\frac{n}{2}\right\rfloor} \frac{(-1)^{r} \alpha^{n-2 r} \beta^{r}}{(n-2 r) !(n-r) ! r !} .
\end{aligned}
$$

Observation 1 It is also useful to note that

$$
\partial_{x}^{m} J_{0}\left(2 \sqrt{\alpha x+\beta x^{2}}\right)=\sum_{n=0}^{\infty} \frac{(n+m) !}{n !} x^{n}{ }_{2} U_{n+m}(\alpha, \beta) .
$$

We have shown that the method we have proposed allows a great deal of flexibility, in the sense that, hardly achievable results with ordinary means, are easily derived within the present computational framework. This is perhaps an indication that the study of these methods should be pursued. 
Remark 1 Before concluding the paper we go back to the auxiliary functions we have defined as, e.g., $c_{0,-\frac{1}{2}}(x)$ in Eq. (17). To better clarify the relevant properties we remind that

$$
e^{-\hat{c} x} \varphi_{0}=C_{0}(x)=\sum_{r=0}^{\infty} \frac{(-x)^{r}}{r !^{2}}, \quad \forall x \in \mathbb{R},
$$

is the Tricomi-Bessel function of zero order [12]. The relevant properties can be easily drawn from Eq. (17) itself. By keeping the derivative with respect to $x$, we find

$$
\partial_{x}^{m} c_{0,-\frac{1}{2}}^{(1)}(x)=(-1)^{m} \int_{0}^{\infty} e^{-s} s^{-\frac{1}{2}+m} \hat{c}^{m} e^{-x s \hat{c}} d s \varphi_{0} .
$$

which suggests the introduction of the function $C_{\mu, v}(x)$ defined as

$$
\begin{aligned}
& C_{\mu, \nu}(x)=\int_{0}^{\infty} e^{-s} s^{v} \hat{c}^{\mu} e^{-x s \hat{c}} d s \varphi_{0}=\int_{0}^{\infty} e^{-s} s^{v} C_{\mu}(x s) d s, \\
& C_{\mu}(x)=\sum_{r=0}^{\infty} \frac{(-x)^{r}}{r ! \Gamma(r+\mu+1)} .
\end{aligned}
$$

In other words Eq. (36) states that $C_{\mu, v}(x)$ can be viewed as Borel $(B-)$ transform $[13,14]$ of the $\mu$-order Tricomi function $C_{\mu}(x)$ [15]. Then, we can get

$$
\partial_{x}^{m} c_{0,-\frac{1}{2}}^{(1)}(x)=(-1)^{m} \int_{0}^{\infty} e^{-s} s^{m-\frac{1}{2}} C_{m}(x s) d s=(-1)^{m} C_{m, m-\frac{1}{2}}(x)
$$

Analogous considerations apply to the functions $J_{\mu, v}^{(2)}(x)$ which can be viewed as the $B$-transform of the Bessel-Wright functions [16].

The $B$-transform has played an important role for the a rigorous formulation of the umbral methods exploited here. Further comments and in particular the link with the Humbert Bessel will be discussed elsewhere.

In this paper we have dealt with definite integrals between $-\infty$ and $\infty$, the umbral method can be exploited to derive definite or indefinite integrals as e.g.,

$$
I_{C}(a)=\int_{0}^{1} C_{0}(a x) d x=\int_{0}^{1} e^{-a \hat{c} x} d x \varphi_{0} \quad \forall a \in \mathbb{R}: a \geq 0
$$

which eventually yields

$$
I_{C}(a)=\frac{1}{\hat{c} a}\left(1-e^{-\hat{c} a}\right) \varphi_{0}=C_{1}(a) .
$$

The presented umbral procedure can be further handled and merged to other techniques of operational nature to obtain further generalizations. It is further worth noting that integration methods based on the umbral procedure does not apply to special function only but to special polynomials as well.

Within such a context the two variable Hermite polynomials

$$
H_{n}(x, y)=n ! \sum_{r=0}^{\left\lfloor\frac{n}{2}\right\rfloor} \frac{x^{n-2 r} y^{r}}{(n-2 r) ! r !}
$$

can be written as an ordinary Newton binomial $[2,6]$

$$
H_{n}(x, y)=\left(x+{ }_{y} \hat{h}\right)^{n} \theta_{0},
$$


where ${ }_{y} \hat{h}$ is an umbral operator acting on the vacuum $\theta_{0}$ in the following way

$$
\begin{aligned}
& \hat{h}^{r} \theta_{0}:=\theta_{r}, \quad \forall r \in \mathbb{R}, \\
& \theta_{r}=\frac{y^{\frac{r}{2}} r !}{\Gamma\left(\frac{r}{2}+1\right)}\left|\cos \left(r \frac{\pi}{2}\right)\right|=\left\{\begin{array}{ll}
0, & (2 s) ! \\
y^{s} \frac{(2 s+1,}{s !}, & r=2 s,
\end{array} \quad \forall s \in \mathbb{Z} .\right.
\end{aligned}
$$

The definite integral

$$
I_{m, n}(x)=\int_{0}^{x} \xi^{m} H_{n}(\xi, y) d \xi
$$

can be then reduced, after a straightforward steps (for further comments see Ref. [11]), to

$$
I_{m, n}(x)=\int_{0}^{x} \xi^{m}(\xi+y \hat{h})^{n} d \xi \theta_{0}=m ! \sum_{s=0}^{\min (n, m+1)}(-1)^{s} \frac{x^{m+s+1}}{(m+s+1) !} \frac{n !}{(n-s) !} H_{n-s}(x, y) .
$$

This result is a straightforward consequence of the (umbral) binomial nature of the Hermite polynomials but can also be obtained with standard means.

In a forthcoming investigation we will see how the merging between umbral methods and negative derivative integration procedures [11] may lead to further interesting developments.

Acknowledgements The work of Dr. S. Licciardi was supported by an Enea Research Center individual fellowship.

Author Contributions Conceptualization: GD; methodology: GD, SL; data curation: SL; validation: GD, BG, SL, MRM; formal analysis: GD, BG, SL, MRM; writing —original draft preparation: GD, SL; writing — review and editing: SL.

Funding Open access funding provided by Ente per le Nuove Tecnologie, l'Energia e l'Ambiente within the CRUI-CARE Agreement.

Open Access This article is licensed under a Creative Commons Attribution 4.0 International License, which permits use, sharing, adaptation, distribution and reproduction in any medium or format, as long as you give appropriate credit to the original author(s) and the source, provide a link to the Creative Commons licence, and indicate if changes were made. The images or other third party material in this article are included in the article's Creative Commons licence, unless indicated otherwise in a credit line to the material. If material is not included in the article's Creative Commons licence and your intended use is not permitted by statutory regulation or exceeds the permitted use, you will need to obtain permission directly from the copyright holder. To view a copy of this licence, visit http://creativecommons.org/licenses/by/4.0/.

\section{References}

1. Dattoli, G., Levi, D., Winternitz, P.: Heisenberg algebra, umbral calculus and orthogonal polynomials. J. Math. Phys. 49, 053509 (2008)

2. Licciardi, S., Pidatella, R.M., Artioli, M., Dattoli, G.: Voigt transform and umbral image. Math. Comput. Appl. 25(3), 49 (2020)

3. Babusci, D., Dattoli, G., Gorska, K., Penson, K.A.: The spherical Bessel and struve functions and operational methods. Appl. Math. Comput. 238, 1-6 (2014)

4. Babusci, D., Dattoli, G., Gorska, K., Penson, K.A.: Symbolic methods for the evaluation of sum rules of Bessel functions. J. Math. Phys. 54, 073501 (2013)

5. Babusci, D., Dattoli, G., Licciardi, S., Sabia, E.: Mathematical Methods for Physicists. World Scientific, Singapore (2019) 
6. Licciardi, S.: PhD Thesis: Umbral Calculus, a Different Mathematical Language, Dep. of Mathematics and Computer Sciences, XXIX cycle, University of Catania, (2018), arXiv:1803.03108 [math.CA]

7. Babusci, D., Dattoli, G.: On Ramanujan Master Theorem, arXiv:1103.3947 [math-ph]

8. Roman, S.: The Umbral Calculus. Dover Publications, New York (2005)

9. Andrews, L.C.: Special Functions For Engineers and Applied mathematicians. Mc Millan, New York (1985)

10. Gradshteyn, I.S., Ryzhik, I.M.: Table of Integrals, Series and Products, Fifth Edition (Allan Jeffrey ed.), Academic Press, (1965)

11. Dattoli, G., Licciardi, S.: Operational, umbral methods, borel transform and negative derivative operator techniques. Int. Transf. Spec. Funct. 31(3), 192-220 (2020)

12. Tricomi, F.G.: Funzioni Speciali, Gheroni, (1959)

13. Dattoli, G., Di Palma, E., Sabia, E., Licciardi, S.: Products of bessel functions and associated polynomials. Appl. Math. Comput. 266(C), 507-514 (2015)

14. Dattoli, G., Di Palma, E., Sabia, E., Gorska, K., Horzela, A., Penson, K.A.: Operational versus umbral methods and the Borel transform. Int. J. Appl. Comput. Math. 3, 3489 (2017)

15. Humbert, P.: Some extensions of Pincherle's polynomials. Proc. Edinburgh Math. Soc. 39, 21-24 (1921)

16. Weisstein, E.W.: Wright function, MathWorld-A Wolfram web resource. http://mathworld. wolfram.com/WrightFunction.html

Publisher's Note Springer Nature remains neutral with regard to jurisdictional claims in published maps and institutional affiliations. 